\title{
Ambiguity of the Moment Tensor
}

\author{
by Jean-Paul Ampuero* and F. A. Dahlen
}

\begin{abstract}
An earthquake on a fault separating two dissimilar materials does not have a well-defined moment density tensor. We present a complete characterization of this bimaterial ambiguity in the general case of slip on a fault in an anisotropic medium. The ambiguity can be eliminated by utilizing a potency density rather than a moment density representation of a bimaterial source.
\end{abstract}

\section{Introduction}

Earthquake seismology is being nourished by a growing body of observational constraints on the structure of fault zones, including geological field studies of exhumed faults (Chester et al., 1993), classical and guided-wave fault-zone tomographic studies (Catchings et al., 2002; Thurber et al., 2003; Ben-Zion, 1998; Li et al., 2000), high-resolution microearthquake relocations (Got et al., 1994; Waldhauser and Ellsworth, 2002), and active fault-zone drilling projects such as the San Andreas Fault Observatory at Depth, the Corinth Rift Laboratory, and the geophysical boreholes into the Nojima Fault Zone (Ohtani et al., 2000). These new constraints are driving fundamental investigations of fault-zone controls on earthquake processes, including both theoretical and observational studies of the dynamics of earthquake rupture on a fault separating two dissimilar materials. Source dynamics on such a bimaterial interface is enriched by the coupling between slip and normal stress, leading naturally to pulselike rupture and directivity (Andrews and Ben-Zion, 1997; Cochard and Rice, 2000). Recently, Rubin and Gillard (2000) and Rubin (2002) observed a pronounced northwestsoutheast asymmetry in the distribution of microearthquake aftershocks along sections of the San Andreas fault that have a strong velocity contrast, up to $20 \%$, across the fault zone. They attributed this along-strike aftershock asymmetry to bimaterial directivity effects. McGuire et al. (2002) have suggested that this mechanism may be a general feature of plate-boundary earthquakes, which may act to enhance the predominance of unilateral rupture on a global scale.

From an observational standpoint, especially when dealing with microseismicity or teleseismic data, the details of earthquake kinematics are usually poorly resolved, and the seismic moment remains one of the few fundamental macroscopic properties of the source that can be reliably estimated from seismograms (Nadeau and Johnson, 1998). The moment tensor has been widely adopted as the preferred phenomenological description of an earthquake in the point-

*Present address: Institute of Geophysics, Seismology, and Geodynamics, ETH Hönggerberg (HPP), CH-8093 Zürich, Switzerland; ampuero @erdw.ethz.ch. source approximation, ever since it was first introduced into seismology by Kostrov (1970) and Gilbert (1971). A shortcoming of this representation is the inherent ambiguity of the surficial moment density tensor in the case of slip on a bimaterial interface. Fundamentally, this ambiguity arises because the scalar moment of an earthquake is defined by $M_{0}=\mu\langle\Delta u\rangle$, where $\mu$ is the rigidity in the vicinity of the source, $\langle\Delta u\rangle$ is the average slip, and $A$ is the fault area (Aki, 1966). In the case of a bimaterial interface with a discontinuity in the rigidity, $\mu^{+} \neq \mu^{-}$, there is no obvious choice for "the" fault rigidity $\mu$, so the earthquake moment $M_{0}$ is not well defined. The reasons for this and other discontinuous source ambiguities have been discussed in a variety of contexts by Woodhouse (1981), Heaton and Heaton (1989), and Ben-Zion $(1989,2001)$. Nevertheless, a recent article by Wu and Chen (2003) suggests that some confusion may still exist regarding this issue in the seismological community. We present a tutorial review of the phenomenological representation of indigenous seismic sources and provide a complete analysis of the moment density ambiguity for earthquakes characterized by slip on a finite bimaterial interface in this article. The analysis allows for the possibility of a general elastic anisotropy but neglects the earth's initial stress, self-gravitation, and rotation, for simplicity.

\section{Strain and Stress Glut}

Let $\mathbf{x}$ be the position vector within an anisotropic elastic medium with mass density $\rho(\mathbf{x})$ and stiffness tensor $C_{i j k l}(\mathbf{x})$. There are only 21 independent components of the fourthorder tensor $C_{i j k l}$, by virtue of the elastic symmetries:

$$
C_{i j k l}=C_{j i k l}=C_{i j l k}=C_{k l i j} .
$$

In the absence of any earthquake source, the equations of motion governing the medium are

$$
\begin{aligned}
& \rho \ddot{u}_{j}=\partial_{i} \sigma_{i j}, \\
& \sigma_{i j}=C_{i j k l} \varepsilon_{k l} .
\end{aligned}
$$


The quantity $u_{j}(\mathbf{x}, t)$ is the infinitesimal displacement of particle $\mathbf{x}$ at time $t$, whereas $\sigma_{i j}$ and $\varepsilon_{k l}=1 / 2\left(\partial_{k} u_{l}+\partial_{l} u_{k}\right)$ are the associated stress and strain; a dot denotes partial differentiation with respect to time, $\partial / \partial t$, and $\partial_{i}$ is shorthand for $\partial / \partial x_{i}$. Equations (2) and (3) must be solved subject to the initial conditions

$$
u_{j}(\mathbf{x}, 0)=0 \quad \dot{u}_{j}(\mathbf{x}, 0)=0,
$$

and to an appropriate boundary condition, stipulating either that there are only outgoing waves at infinity or that there is no traction on the free surface of a finite earth model. Backus and Mulcahy (1976a) made the elementary but profound observation that the unique solution of equations (2-4) is an eternally quiescent and, therefore, seismologically uninteresting earth: $u_{j}(\mathbf{x}, t)=0$ at all positions $\mathbf{x}$ and for all times $t$. Newton's second law $\rho \ddot{u}_{j}=\partial_{i} \sigma_{i j}$ is a genuine law of mechanics, so an indigenous seismic source must be due to a breakdown of Hooke's constitutive "law" $\sigma_{i j}=C_{i j k l} \varepsilon_{k l}$.

Generalizing the description of a static transformational phase change introduced by Eshelby (1957), we may represent a source phenomenologically by a specified stressfree strain, denoted by $\varepsilon_{k l}^{*}(\mathbf{x}, t)$. The stress-strain constitutive relation $\sigma_{i j}=C_{i j k l} \varepsilon_{k l}$ is replaced by

$$
\sigma_{i j}=C_{i j k l}\left(\varepsilon_{k l}-\varepsilon_{k l}^{*}\right),
$$

where it is assumed that $\varepsilon_{k l}^{*}$ is nonzero only for $t \geq 0$, and only inside some nonelastic source region $V$, within which Hooke's "law" is violated. The quantity

$$
\sigma_{i j}^{*}=C_{i j k l} \varepsilon_{k l}^{*}
$$

is the stress glut within the source region $V$ (Backus and Mulcahy, 1976a); by analogy, we may alternatively refer to the stress-free strain $\varepsilon_{k l}^{*}$ as the strain glut. On inserting equation (5) into equation (2), we may write the equation of motion in an inhomogeneous form that allows for the possibility of an earthquake, namely,

$$
\rho \ddot{u}_{j}=\partial_{i}\left(C_{i j k l} \varepsilon_{k l}\right)+f_{j}^{*} .
$$

The quantity

$$
f_{j}^{*}=-\partial_{i} \sigma_{i j}^{*}=-\partial_{i}\left(C_{i j k l} \varepsilon_{k l}^{*}\right)
$$

is the equivalent body force, which gives rise to the same response $u_{j}$ as the earthquake. We assume, for the moment, that both the stiffness tensor $C_{i j k l}$ and the strain glut $\varepsilon_{k l}^{*}$ are smooth functions of position $\mathbf{x}$ within the source region $V$, so that the derivative $\partial_{i}$ in equation (8) is well defined. We also assume, for simplicity, that the source region $V$ is buried within the earth, so that we need not be concerned with an equivalent surface force in addition to the equivalent body force (Backus and Mulcahy, 1976a).

\section{Displacement, Strain, and Stress Green Tensors}

Following Burridge and Knopoff (1964) and Aki and Richards (2002, section 2.4) we write the displacement Green tensor of the medium in the form $G_{q n}(\mathbf{r}, t ; \mathbf{s}, \tau)$. By definition, $G_{q n}(\mathbf{r}, t ; \mathbf{s}, \tau)$ is the $q$ th component of the displacement at a receiver point $\mathbf{r}$ and at time $t$, due to an impulsive force,

$$
f_{j}(\mathbf{x}, t)=\delta_{j n} \delta(\mathbf{x}-\mathbf{s}) \delta(t-\tau),
$$

applied in the $n$th direction at a source point $\mathbf{s}$ and at time $\tau$. We denote the $p q$ th component of strain and the $i j$ th component of stress at point $\mathbf{r}$ and time $t$ due to the applied force (9) by

$$
\begin{gathered}
E_{p q n}(\mathbf{r}, t ; \mathbf{s}, \tau)=\frac{1}{2}\left[\frac{\partial G_{q n}(\mathbf{r}, t ; \mathbf{s}, \tau)}{\partial r_{p}}+\frac{\partial G_{p n}(\mathbf{r}, t ; \mathbf{s}, \tau)}{\partial r_{q}}\right], \\
T_{i j n}(\mathbf{r}, t ; \mathbf{s}, \tau)=C_{i j p q}(\mathbf{r}) E_{p q n}(\mathbf{r}, t ; \mathbf{s}, \tau) .
\end{gathered}
$$

These associated strain and stress Green tensors are symmetric in the customary sense $E_{p q n}=E_{q p n}$ and $T_{i j n}=T_{j i n}$.

In addition to differentiating the displacement Green tensor with respect to the receiver coordinates $\mathbf{r}$, as in equation (10), we can differentiate it with respect to the source coordinates $\mathbf{s}$. Anticipating the reciprocity relation (equation [15]) we introduce the symmetrized source derivative

$$
E_{n p q}(\mathbf{r}, t ; \mathbf{s}, \tau)=\frac{1}{2}\left[\frac{\partial G_{n q}(\mathbf{r}, t ; \mathbf{s}, \tau)}{\partial s_{p}}+\frac{\partial G_{n p}(\mathbf{r}, t ; \mathbf{s}, \tau)}{\partial s_{q}}\right]
$$

which can be interpreted as the $n$th component of the displacement at a receiver point $\mathbf{r}$ and at time $t$, due to a doublecouple body force

$$
\begin{aligned}
f_{j}(\mathbf{x}, t)=-\frac{1}{2} \delta_{j q} \partial_{p} \delta(\mathbf{x} & -\mathbf{s}) \delta(t-\tau) \\
& -\frac{1}{2} \delta_{j p} \partial_{q} \delta(\mathbf{x}-\mathbf{s}) \delta(t-\tau)
\end{aligned}
$$

applied at a source point $\mathbf{s}$ and at time $\tau$. The symmetry $E_{n p q}=E_{n q p}$ is associated with the indistinguishability of the $p$ th and $q$ th directions of the double couple (13).

The principle of source-receiver reciprocity stipulates that

$$
G_{q n}(\mathbf{r}, t ; \mathbf{s}, \tau)=G_{n q}(\mathbf{s}, t ; \mathbf{r}, \tau) .
$$

The $n$th and $q$ th directions must be interchanged, in addition to the locations of the source $\mathbf{s}$ and receiver $\mathbf{r}$ (Aki and Richards, 2002, equation 2.39). The Green strains (10) and (12) satisfy an analogous reciprocity relation, namely,

$$
E_{p q n}(\mathbf{r}, t ; \mathbf{s}, \tau)=E_{n p q}(\mathbf{s}, t ; \mathbf{r}, \tau)
$$


No reciprocity relation analogous to (14) or (15) involves the stress tensors $T_{i j n}(\mathbf{r}, t ; \mathbf{s}, \tau)=C_{i j p q}(\mathbf{r}) E_{p q n}(\mathbf{r}, t ; \mathbf{s}, \tau)$ and $T_{n i j}(\mathbf{s}, t ; \mathbf{r}, \tau)=C_{i j p q}(\mathbf{s}) E_{n p q}(\mathbf{s}, t ; \mathbf{r}, \tau)$, because the former involves the stiffness $C_{i j p q}$ at the receiver $\mathbf{r}$, whereas the latter involves $C_{i j p q}$ at the source $\mathbf{s}$. We shall assume that the displacement, strain, and stress Green tensors are available for the elastic medium under consideration; they may be computed using a variety of numerical techniques, including normal-mode summation (Dahlen and Tromp, 1998, section 4.1.7) or the spectral element method (Komatitsch and Vilotte, 1998; Komatitsch and Tromp, 1999, 2002a, 2002b).

\section{Response to a Smooth Strain-Glut Source}

The Green tensors can be used, in conjunction with the principles of superposition and causality, to represent the response of the medium to more general phenomenologically prescribed forces. Specifically, we can express the displacement response $u_{n}(\mathbf{r}, t)$ to a smoothly varying imposed body force $f_{j}(\mathbf{x}, t)$ within a source region $V$ in the form

$$
u_{n}(\mathbf{r}, t)=\int_{0}^{t} d \tau \iiint_{V} G_{n j}(\mathbf{r}, t ; \mathbf{x}, \tau) f_{j}(\mathbf{x}, \tau) d^{3} \mathbf{x} .
$$

On inserting the strain-glut-equivalent body force equation (8) into equation (16) and integrating by parts, we obtain

$u_{n}(\mathbf{r}, t)=\int_{0}^{t} d \tau \iiint_{V} E_{n i j}(\mathbf{r}, t ; \mathbf{x}, \tau) C_{i j k l}(\mathbf{x}) \varepsilon_{k l}^{*}(\mathbf{x}, \tau) d^{3} \mathbf{x}$,

where we have assumed that $\varepsilon_{k l}^{*}(\mathbf{x}, \tau)$ goes to zero smoothly outside the source region $V$, to eliminate the integral over the boundary $\partial V$. The first of the stiffness symmetries (1) has been used to express the result (17) in terms of the symmetrized derivative $E_{n i j}(\mathbf{r}, t ; \mathbf{x}, \tau)$. On associating the stiffness tensor $C_{i j k l}(\mathbf{x})$ with $\varepsilon_{k l}^{*}(\mathbf{x}, \tau)$ and making the identification in equation (6), we can rewrite equation (17) in the form

$$
u_{n}(\mathbf{r}, t)=\int_{0}^{t} d \tau \iiint_{V} E_{n i j}(\mathbf{r}, t ; \mathbf{x}, \tau) \sigma_{i j}^{*}(\mathbf{x}, \tau) d^{3} \mathbf{x} .
$$

Equation (18) expresses $u_{n}(\mathbf{r}, t)$ as the response to a spacetime superposition of double couples, weighted by the stress glut $\sigma_{i j}^{*}(\mathbf{x}, \tau)$.

Alternatively, we can invoke the reciprocity relation (15) in equation (17), and associate the stiffness tensor $C_{i j k l}(\mathbf{x})=C_{k l i j}(\mathbf{x})$ with the Green strain $E_{i j n}(\mathbf{x}, t ; \mathbf{r}, \tau)$, to obtain

$$
u_{n}(\mathbf{r}, t)=\int_{0}^{t} d \tau \iiint_{V} T_{k l n}(\mathbf{x}, t ; \mathbf{r}, \tau) \varepsilon_{k l}^{*}(\mathbf{x}, \tau) d^{3} \mathbf{x} .
$$

Equation (19) expresses $u_{n}(\mathbf{r}, t)$ in terms of the strain glut $\varepsilon_{k l}^{*}(\mathbf{x}, \tau)$ rather than the stress glut $\sigma_{i j}^{*}(\mathbf{x}, \tau)$. The associated Green tensor in this case is the $k l$ th component of the stress at the source point $\mathbf{x}$ and at time $t$, due to an impulsive force exerted in the $n$th direction at the receiver $\mathbf{r}$ and at time $\tau$. A more seismologically familiar interpretation of the representation (19) in terms of forces or couples situated in the source region is precluded by the absence of a reciprocity relation for the Green stresses, $T_{i j n}(\mathbf{r}, t ; \mathbf{s}, \tau) \neq T_{n i j}(\mathbf{s}, t ; \mathbf{r}, \tau)$.

\section{Slip on an Ideal Fault}

Thus far, we have considered a source specified by a smoothly varying strain glut $\varepsilon_{k l}^{*}$ within a three-dimensional source volume $V$. Suppose instead that the source region is a two-dimensional fault surface $\Sigma$; let $\xi$ denote the position of points on the surface, and let $\hat{\mathbf{n}}(\xi)$ be the unit normal to the fault. The side toward which the normal points is referred to as the plus or front side of the fault surface, whereas the other side is referred to as the minus or back side. For any function $q(\xi)$ that is discontinuous across $\Sigma$, we let

$$
q^{ \pm}(\xi)=\lim _{h \rightarrow 0} q(\xi \pm h \hat{\mathbf{n}})
$$

denote the values at juxtaposed points on either side. The slip $\Delta u_{k}(\xi, \tau)$ of the front side relative to the back side is then

$$
\Delta u_{k}=u_{k}^{+}-u_{k}^{-} .
$$

We denote the magnitude of the slip vector by $\Delta u(\xi, \tau)$ and we denote its instantaneous direction by $e_{k}(\xi, \tau)$, so that

$$
\Delta u_{k}=\Delta u e_{k} .
$$

The fault may be a bimaterial interface, with different physical properties, $\rho^{ \pm}(\xi)$ and $C_{i j k l}^{ \pm}(\xi)$, on either side.

An ideal fault is one that can be completely characterized by such a kinematically prescribed tangential slip distribution $\Delta u_{k}(\xi, \tau)$. The earth model is assumed to be perfectly elastic everywhere except on the fault surface $\Sigma$. The breakdown of Hooke's "law" is confined to the fault; the strain glut is a singular distribution, given explicitly by (Backus and Mulcahy, 1976b)

$$
\varepsilon_{k l}^{*}(\mathbf{x}, \tau)=\iint_{\Sigma} p_{k l}(\xi, \tau) \delta(\mathbf{x}-\xi) d^{2} \xi
$$

where

$$
p_{k l}=\frac{1}{2} \Delta u\left(n_{k} e_{l}+n_{l} e_{k}\right) .
$$


Ben-Menahem and Singh (1981), Heaton and Heaton (1989), and Ben-Zion $(1989,2001)$ refer to the product of the average slip on a fault and the fault area, $\langle\Delta u\rangle A$, as the earthquake potency. We are unable to suggest a more appropriate or expressive term, so we shall (reluctantly) refer to $p_{k l}(\xi, \tau)=p_{l k}(\xi, \tau)$ as the potency density tensor.

Equation (19) was derived under the assumption that the strain glut $\varepsilon_{k l}^{*}(\mathbf{x}, \tau)$ is smooth and nonsingular; however, it is valid for a singular strain glut as well, provided that the products and integrations are properly interpreted in the sense of distributions. On inserting the representation (23), we can express the response to a specified fault slip in the form

$$
u_{n}(\mathbf{r}, t)=\int_{0}^{t} d \tau \iint_{\Sigma} T_{k l n}^{ \pm}(\xi, t ; \mathbf{r}, \tau) p_{k l}(\xi, \tau) d^{2} \xi
$$

The tractions $n_{k} T_{k l n}$ and $n_{l} T_{k l n}$ are continuous across the fault surface $\Sigma$, so it is immaterial whether the Green stress $T_{k l n}$ in this representation is evaluated on the front side or the back side. We have written equation (25) in terms of $T_{k l n}^{ \pm}$to emphasize this immateriality.

We have obtained the preceding result by recognizing that a fault-slip source has an associated singular strain glut, given by equation (23); however, it is also possible to derive equation (25) without recourse to distribution theory, by means of a more classical argument based on the Volterra representation theorem. In fact, it follows immediately from equation (3.2) of Aki and Richards (2002), by relabeling $\mathbf{x} \rightarrow \mathbf{r}$, invoking the principle of source-receiver reciprocity, $G_{n p}(\mathbf{r}, t ; \xi, \tau)=G_{p n}(\xi, t ; \mathbf{r}, \tau)$, and recognizing the product $C_{i j p q}(\xi) \partial G_{p n}(\xi, t ; \mathbf{r}, \tau) / \partial \xi_{\mathrm{q}}$ as the Green stress $T_{i j n}(\xi, t ; \mathbf{r}, \tau)$. In the classical derivation it is abundantly clear that the result (25) is applicable to a bimaterial interface, with $\rho^{+} \neq \rho^{-}$ and $C_{i j k l}^{+} \neq C_{i j k l}^{-}$. In our alternative distribution theory derivation, the applicability to a bimaterial interface is guaranteed by the continuity of the Green tractions $n_{k} T_{k l n}$ and $n_{l} T_{k l n}$. Upon inserting the singular strain-glut representation (23) into equation (19), we are never confronted with the product of a Dirac delta distribution and a Heaviside step function, which is undefined.

The potency density representation (25) of the response $u_{n}(\mathbf{r}, t)$ to a prescribed slip $\Delta u_{k}(\xi, \tau)$ on a possibly bimaterial interface is more useful for many computational purposes than the representation advocated by Ben-Zion (1989), which is of the form

$$
u_{n}(\mathbf{r}, t)=\int_{0}^{t} d \tau \iint_{\Sigma} B_{n k}(\mathbf{r}, t ; \xi, \tau) \Delta u_{k}(\xi, \tau) d^{2} \xi .
$$

The quantity $B_{n k}(\mathbf{r}, t ; \xi, \tau)$ in equation (26) is, by definition, the $n$th component of the displacement response at a receiver point $\mathbf{r}$ and time $t$, due to a unit point dislocation in the $k$ th direction at a point $\xi$ and time $\tau$ on the fault plane. Analytical expressions for $B_{n k}(\mathbf{r}, t ; \xi, \tau)$ in the special case of slip $\Delta u_{k}(\xi$, $\tau)$ on a fault separating two dissimilar, isotropic half-spaces are given by Ben-Zion (1990, 1999). In general, numerical computation is needed; however, the dislocation response $B_{n k}(\mathbf{r}, t ; \xi, \tau)$ cannot be computed using conventional wave propagation codes without the introduction of "split nodes" on the fault. In contrast, all that is needed to compute the Green kernel $T_{k l n}^{ \pm}(\xi, t ; \mathbf{r}, \tau)$ in equation (25) is the synthetic stress history at the nodes on the fault plane, due to impulsive sources situated at the receivers $\mathbf{r}$.

\section{Ambiguity of the Moment Density Tensor}

The integrand in the unambiguous potency density representation (25) can be manipulated as follows:

$$
\begin{aligned}
T_{k l n}^{ \pm} p_{k l} & =C_{k l i j}^{ \pm} E_{i j n}^{ \pm} p_{k l} \\
& =E_{i j n}^{ \pm} C_{i j k l}^{ \pm} p_{k l} \\
& =E_{n i j}^{ \pm} C_{i j k l}^{ \pm} p_{k l} .
\end{aligned}
$$

The Green tensors $E_{n i j}^{ \pm}(\mathbf{r}, t ; \xi, \tau)$ obtained by utilizing the reciprocity relation (15) in the final line of equation (27) are the displacement responses at point $\mathbf{r}$ and time $t$, due to double couples,

$$
\begin{aligned}
f_{k}^{ \pm}(\mathbf{x}, t)=-\frac{1}{2} & \delta_{k j} \partial_{i} \delta^{ \pm}(\mathbf{x}-\xi) \delta(t-\tau) \\
& -\frac{1}{2} \delta_{k i} \partial_{j} \delta^{ \pm}(\mathbf{x}-\xi) \delta(t-\tau)
\end{aligned}
$$

situated at adjacent points on either side of the fault. The front-side and back-side Dirac delta functions in equation (28) are defined by limiting relations analogous to those for a nonsingular but discontinuous function in equation (20):

$$
\delta^{ \pm}(\mathbf{x}-\xi)=\lim _{h \rightarrow 0} \delta(\mathbf{x}-(\xi \pm \mathrm{h} \hat{\mathbf{n}})) .
$$

The stiffness factors $C_{i j k l}^{ \pm}(\xi)$ in the final line of equation (27) can be associated with the tensor $\left.p_{k l} \xi, \tau\right)$ to form front-side and back-side moment density tensors,

$$
m_{i j}^{ \pm}=C_{i j k l}^{ \pm} p_{k l} .
$$

Equation (25) can be rewritten in terms of these tensors in the form

$$
u_{n}(\mathbf{r}, t)=\int_{0}^{t} d \tau \iint_{\Sigma} E_{n i j}^{ \pm}(\mathbf{r}, t ; \xi, \tau) m_{i j}^{ \pm}(\xi, \tau) d^{2} \xi .
$$

The result (31) stipulates that the prescribed fault slip $\Delta u_{k}(\xi, \tau)$ is equivalent either to a superposition of double couples situated on the front side of the fault, with moment density $m_{k l}^{+}(\xi, \tau)$, or to a superposition of double couples situated on the back side of the fault, with moment density 
$m_{k l}^{-}(\xi, \tau)$. This illustrates the fundamental ambiguity of the moment density tensor in a bimaterial interface, with a contrast in elastic stiffness, $C_{i j k l}^{+} \neq C_{i j k l}^{-}$. Observed seismograms $u_{n}(\mathbf{r}, t)$ can either be inverted for the front-side moment density tensor $m_{k l}^{+}$using $E_{n k l}^{+}$as the Green tensor, or they can be inverted for the back-side moment density tensor $m_{k l}^{-}$using $E_{n k l}^{-}$as the Green tensor. The resulting moment density tensors are obviously different; nevertheless, they produce an identical response (31) at every receiver $\mathbf{r}$ and for all times $t$.

If the stiffness is continuous across the fault, then the Green strain will be continuous also, $E_{n k l}^{+}=E_{n k l}^{-}$. Only in that case is there a unique, unambiguous moment density tensor,

$$
m_{i j}=C_{i j k l} p_{k l},
$$

where $C_{i j k l}=C_{i j k l}^{+}=C_{i j k l}^{-}$is the stiffness. The singular stress glut

$$
\sigma_{i j}^{*}(\mathbf{x}, \tau)=\iint_{\Sigma} m_{i j}(\xi, \tau) \delta(\mathbf{x}-\xi) d^{2} \xi
$$

and associated equivalent body force

$$
f_{j}^{*}(\mathbf{x}, \tau)=-\iint_{\Sigma} m_{i j}(\xi, \tau) \partial_{i} \delta(\mathbf{x}-\xi) d^{2} \xi
$$

are then also both well defined.

\section{Characterization of the Ambiguity}

More generally, as we shall show in this section, it is possible to rewrite equation (31) in the discontinuous case, $C_{i j k l}^{+} \neq C_{i j k l}^{-}$, in the form

$$
u_{n}(\mathbf{r}, t)=\int_{0}^{t} d \tau \iint_{\Sigma} E_{n i j}^{\gamma}(\mathbf{r}, t ; \xi, \tau) m_{i j}^{\gamma}(\xi, \tau) d^{2} \xi,
$$

where $E_{n i j}^{\gamma}(\mathbf{r}, t ; \xi, \tau)$ is a weighted linear combination of the front-side and back-side Green strains, of the form

$$
E_{n i j}^{\gamma}=\gamma E_{n i j}^{+}+(1-\gamma) E_{n i j}^{-}, \quad 0 \leq \gamma \leq 1 .
$$

The quantity $\gamma$ is a parameter specifying the fraction of the strain $E_{n i j}^{\gamma}(\mathbf{r}, t ; \xi, \tau)$ associated with the front side of the fault. No summation over $\gamma$ is implied in equation (36), or in other equations containing products of $\gamma$-dependent quantities in what follows.

To find the quantities $m_{i j}^{\gamma}(\xi, \tau), 0 \leq \gamma \leq 1$ in the representation (35), we make use of the compliance tensor, $S_{k l i j}$, which relates the elastic strain $\varepsilon_{k l}$ to the stress $\sigma_{i j}$, rather than vice versa:

$$
\sigma_{i j}=C_{i j k l} \varepsilon_{k l} \Leftrightarrow \varepsilon_{k l}=S_{k l i j} \sigma_{i j} .
$$

The invertibility (37) of the stress-strain relation is guaranteed by the positive definite character of both the stiffness and compliance tensors:

$$
\begin{gathered}
\varepsilon_{i j} C_{i j k l} \varepsilon_{k l}>0 \text { for all } \varepsilon_{i j} \neq 0, \\
\sigma_{k l} S_{k l i j} \sigma_{i j}>0 \text { for all } \sigma_{k l} \neq 0,
\end{gathered}
$$

There are only 21 independent components $S_{k l i j}$, by virtue of the compliance symmetries, analogous to the stiffness symmetries in equation (1),

$$
S_{k l i j}=S_{l k i j}=S_{k l j i}=S_{i j k l} .
$$

The stiffness and compliance tensors of a general anisotropic medium are related by $C_{i j p q} S_{p q k l}=1 / 2\left(\delta_{i k} \delta_{j l}+\delta_{i l} \delta_{j k}\right)$.

As we shall now demonstrate, the $\gamma$-dependent moment density tensors $m_{i j}^{\gamma}(\xi, \tau)$ in the representation (35) are defined implicitly by

$$
p_{k l}=S_{k l i j}^{\gamma} m_{i j}^{\gamma}
$$

where $S_{k l i j}^{\gamma}(\xi)$ is a weighted linear combination of the frontside and back-side compliances,

$$
S_{k l i j}^{l}=\gamma S_{k l i j}^{+}+(1-\gamma) S_{k l i j}^{-}, \quad 0 \leq \gamma \leq 1,
$$

analogous to the weighted Green strains $E_{n i j}^{\gamma}(\mathbf{r}, t ; \xi, \tau)$ defined in equation (36). To verify that the definition (41) leads to the ambiguous $0 \leq \gamma \leq 1$ representation (35), we return to the unambiguous representation (25) and manipulate the integrand in the following manner:

$$
\begin{aligned}
T_{k l n}^{ \pm} p_{k l} & =T_{k l n}^{ \pm} S_{k l i j}^{\gamma} m_{i j}^{\gamma} \\
& =T_{k l n}^{ \pm} S_{i j k l}^{\gamma} m_{i j}^{\gamma} \\
& =T_{k l n}^{ \pm}\left[\gamma S_{i j k l}^{+}+(1-\gamma) S_{i j k l}^{-}\right] m_{i j}^{\gamma} \\
& =\left[\gamma S_{i j k l}^{+} T_{k l n}^{+}+(1-\gamma) S_{i j k l j}^{-} T_{k l n}^{-}\right] m_{i j}^{\gamma} \\
& =\left[\gamma E_{i j n}^{+}+(1-\gamma) E_{i j n}^{-}\right] m_{i j}^{\gamma} \\
& =\left[\gamma E_{n i j}^{+}+(1-\gamma) E_{n i j}^{-i}\right] m_{i j}^{\gamma} \\
& =E_{n i j}^{\gamma} m_{i j}^{\gamma} .
\end{aligned}
$$

This step-by-step argument confirms that the displacement $u_{n}(\mathbf{r}, t)$ can be written in the form (35), with $m_{i j}^{\gamma}(\xi, \tau)$ defined implicitly by equation (41).

The defining relation can be inverted in a manner analogous to (37), to find the moment density tensor $m_{i j}^{\gamma}(\xi, \tau)$ explicitly in terms of the potency density tensor $p_{k l}(\xi, \tau)$, rather than vice versa:

$$
p_{k l}=S_{k l i j}^{\gamma} m_{i j}^{\gamma} \Leftrightarrow m_{i j}^{\gamma}=C_{i j k l}^{\gamma} p_{k l} .
$$

The physically appealing restriction of the weighting parameter $\gamma$ to the range $0 \leq \gamma \leq 1$ guarantees the positive defi- 
niteness of $S_{i j k l}^{\gamma}$, and thus the invertibility (44), for arbitrary material contrasts, $S_{k l i j}^{+} \neq S_{k l i j}^{-}$and $C_{i j k l}^{+} \neq C_{i j k l}^{-}$. The dependence of the compliance $S_{k l i j}^{\prime}$ on the parameter $0 \leq \gamma \leq 1$ is a simple linear combination (42), corresponding to $\gamma$ percent of the stiffness on the front side and $1-\gamma$ percent on the back side of the fault. The inversion (44) will "scramble" the $\gamma$ dependence, so that neither the $\gamma$-dependent stiffness $C_{i j k l}^{\gamma}$ nor the $\gamma$-dependent moment density $m_{i j}^{\gamma}=C_{i j k l}^{\gamma} p_{k l}$ will be such a simple linear combination of the front-side and back-side tensors $C_{i j k l}^{ \pm}$and $m_{i j}^{ \pm}$.

The relations (35), (36), (42), and (44) completely encapsulate the ambiguity of the bimaterial moment density tensor. Any choice of the stiffness weighting parameter $0 \leq$ $\gamma \leq 1$ is permissible, and every choice leads to a different moment density tensor $m_{i j}^{\gamma}(\xi, \tau)$. If we wish to invert observed seismograms $u_{n}(\mathbf{r}, t)$ for a particular $m_{i j}^{\gamma}(\xi, \tau)$, then it is necessary to use a Green tensor $E_{n i j}^{\gamma}(\mathbf{r}, t ; \xi, \tau)$ corresponding to a superposition of double couples,

$$
f_{k}^{\prime}(\mathbf{x}, \tau)=\gamma f_{k}^{+}(\mathbf{x}, \tau)+(1-\gamma) f_{k}^{-}(\mathbf{x}, \tau),
$$

that are situated $\gamma$ percent on the front side and $1-\gamma$ percent on the back side of the fault. If $\gamma=0$ the couples are entirely situated on the back side, and their moment density is $m_{i j}^{-}=C_{i j k l}^{-} p_{k l}$, whereas if $\gamma=1$ they are situated on the front side, and their moment density is $m_{i j}^{+}=C_{i j k l}^{+} p_{k l}$. These two limiting cases represent the binary $m_{i j}^{ \pm}$ambiguity that is inherent in equation (31); we see, however, that there is actually a continuum of ambiguity associated with the continuum of choices $0 \leq \gamma \leq 1$ for "the" fault stiffness tensor $C_{i j k l}^{\gamma}$.

In summary, the response $u_{n}(\mathbf{r}, t)$ to a specified slip $\Delta u_{k}(\xi, \tau)$ is identical with the motion produced by an infinite suite of possible body forces:

$$
f_{j}^{* \gamma}(\mathbf{x}, \tau)=-\iint_{\Sigma} m_{i j}^{\gamma}(\xi, \tau) \partial_{i} \delta^{\gamma}(\mathbf{x}-\xi) d^{2} \xi, \quad 0 \leq \gamma \leq 1,
$$

where

$$
\delta^{\gamma}(\mathbf{x}-\xi)=\gamma \delta^{+}(\mathbf{x}-\xi)+(1-\gamma) \delta^{-}(\mathbf{x}-\xi)
$$

is a weighted linear combination of front-side and back-side Dirac delta functions. The ambiguous equivalent body force (46) is the generalization of equation (34) to the case of slip on a bimaterial interface.

\section{Shear Fault in an Isotropic Medium}

The preceding results are simplified in an isotropic elastic medium, with stiffness and compliance tensors of the form

$$
C_{i j k l}=\left(\kappa-\frac{2}{3} \mu\right) \delta_{i j} \delta_{k l}+\mu\left(\delta_{i k} \delta_{j l}+\delta_{i l} \delta_{j k}\right),
$$

$$
S_{k l i j}=\left(\frac{1}{9 \kappa}-\frac{1}{6 \mu}\right) \delta_{k l} \delta_{i j}+\frac{1}{4 \mu}\left(\delta_{k i} \delta_{l j}+\delta_{k j} \delta_{l i}\right),
$$

where $\kappa(\mathbf{x})$ and $\mu(\mathbf{x})$ are the incompressibility and rigidity, respectively. For the first time in this article, we shall also restrict attention to a fault whose walls are not allowed to open or interpenetrate, that is, we shall assume that the slip is purely tangential, so that

$$
n_{k} e_{k}=0
$$

The front-side and back-side moment density tensors (30) reduce in that case to

$$
m_{i j}^{ \pm}=\mu^{ \pm} \Delta u\left(n_{i} e_{j}+n_{j} e_{i}\right),
$$

where the tangency condition (50) eliminates any dependence on the incompressibility. More generally, upon substituting equation (48) into the relation $p_{k l}=S_{k l i j}^{\gamma} m_{i j}^{\gamma}$, and inverting to find the $\gamma$-dependent moment density tensor, we obtain

$$
m_{i j}^{\gamma}=\mu^{\gamma} \Delta u\left(n_{i} e_{j}+n_{j} e_{i}\right)
$$

where

$$
\frac{1}{\mu^{\gamma}}=\frac{\gamma}{\mu^{+}}+\frac{1-\gamma}{\mu^{-}} \text {or } \mu^{\gamma}=\frac{\mu^{+} \mu^{-}}{\gamma \mu^{-}+(1-\gamma) \mu^{+}} .
$$

Equations (52) and (53) characterize the moment density ambiguity in the case of a prescribed tangential slip $\Delta u_{k}(\xi$, $\tau)$ on a finite bimaterial interface in an isotropic medium. As in the anisotropic case, every choice of the weighting parameter $0 \leq \gamma \leq 1$ gives rise to a different fault rigidity $\mu^{\gamma}(\xi)$ and a different moment density tensor $m_{i j}^{\gamma}(\xi, \tau)$. As before, every such choice is associated with a different equivalent body force (46).

Wu and Chen (2003) advocate defining "the" moment density tensor at a bimaterial interface by

$$
m_{i j}=\mu \Delta u\left(n_{i} e_{j}+n_{j} e_{i}\right)
$$

where

$$
\mu=\frac{2 \mu^{+} \mu^{-}}{\mu^{+}+\mu^{-}} .
$$

Comparing equation (55) with equation (53), we see that their definition corresponds to the choice $\gamma=0.5$. This is a permissible choice; however, there is nothing special or unique about it, as Wu and Chen (2003) assert. Moreover, if one makes their choice and seeks to determine $m_{i j}^{0.5}$ by inversion of observed seismograms, then it is necessary to 
use an equivalent body force $f_{j}^{* 0.5}$ and associated Green strain $E_{n i j}^{0.5}$ corresponding to a superposition of double couples that is $50 \%$ on one side of the fault and $50 \%$ on the other.

It could be argued that the choice $\gamma=0.5$ is appealing on the grounds of simplicity: the expression (55) is, after all, a symmetric definition of "the" fault rigidity, in which the front-side and back-side values, $\mu^{+}$and $\mu^{-}$, play equal roles. However, other choices also lead to simple, symmetric definitions; for instance,

$$
\mu^{\gamma}=\frac{1}{2}\left(\mu^{+}+\mu^{-}\right) \text {when } \gamma=\frac{\mu^{+}}{\mu^{+}+\mu^{-}} .
$$

The choice $\gamma=0.5$ of $\mathrm{Wu}$ and Chen (2003) is also reminiscent of the partitioning of static slip in an antiplane shear crack in an isotropic medium. In fact, it can be shown, using the spectral fault stiffness formalism (Andrews, 1980; Ampuero et al., 2002), that the static displacements $u^{ \pm}(\xi)$ on either side of such a mode III crack are related to the static slip $\Delta u(\xi)$ by $u^{ \pm}= \pm \mu^{\mp} \Delta u /\left(\mu^{+}+\mu^{-}\right)$. When combined with equations (54) and (55), this enables us to express the static seismic moment in the form $M_{0}^{* 0.5}=\mu^{+}\left\langle u^{+}\right\rangle A-$ $\mu^{-}\left\langle u^{-}\right\rangle A$, which can be viewed as an equally weighted sum of front-side and back-side "partial" moments, $M_{0}^{* 0.5}=$ $\mu^{+}\left\langle u^{+}-0\right\rangle A+\mu^{-}\left\langle 0-u^{-}\right\rangle A$. However, such an interpretation does not carry over to static inplane (mode II) faulting, for which the relation between $u^{ \pm}(\xi)$ and $\Delta u(\xi)$ involves Poisson's ratio as well as the rigidity. Moreover, in the general time-dependent case, the front-side and back-side displacements $u^{ \pm}(\xi, \tau)$ are related to the slip $\Delta u(\xi, \tau)$ by a nonlocal space-time convolution.

Physical considerations likewise fail to provide any guidance regarding the apportionment of the stress glut onto one side of a fault or the other. The definition of a surficial moment density underlies a macroscopic representation of very complex fault zone processes, by lumping volumedistributed anelasticity onto a nominal fault plane. However, relatively little is known about off-fault dynamic processes that could guide a physically based choice of the parameter $\gamma$. It is clear that the potential for dynamic secondary faulting and damage around a mode II propagating rupture is not symmetric: dynamic microcracking, which may contribute to the radiated wave field, is more intensive on the dilational side of the main crack than on the compressive side (Yamashita, 2000; Poliakov et al., 2002). Anelasticity of a gouge zone may also contribute to the seismic moment, with the parameter $\gamma$ being related to the relative location of the main slip plane, or localization band, inside the gouge layer. It is often observed that deformation localizes at the boundary of the gouge zone (Chambon et al., 2002). Likewise, elastic deformation of an unmodeled low-velocity fault zone may contribute to the apparent seismic moment as an equivalent inclusion in the sense of Eshelby (1957) and Mura (1982). It is also likely that some ruptures prefer to run along the boundary of a low-velocity layer rather than cutting through the middle of the fault zone (Brietzke and Ben-Zion, 2003). These observations would suggest that either $\gamma=0$ or $\gamma=1$.

In summary, there is no compelling argument, either theoretical or physical, for preferring any particular choice of the moment density tensor $m_{i j}^{\gamma}(\xi, \tau), 0 \leq \gamma \leq 1$, over any other. This ambiguity does not exist when the source is represented by its potency density tensor $p_{k l}(\xi, \tau)$.

\section{Conclusion}

The moment density tensor $m_{i j}(\xi, \tau)$ associated with a specified slip distribution $\Delta u_{k}(\xi, \tau)$ on a bimaterial interface is fundamentally ambiguous, as Heaton and Heaton (1989) and Ben-Zion $(1989,2001)$ have clearly noted. In fact, such a bimaterial slip source has an infinite number of possible moment densities $m_{i j}^{\gamma}(\xi, \tau)$, where $0 \leq \gamma \leq 1$ is a measure of the extent to which the source is considered to lie on one side of the fault or other, in a sense made precise in this article. If, as usual, a surficial seismic moment representation (slip on a fault plane) is adopted, the parameter $\gamma$ cannot be inverted from seismological data but must be arbitrarily fixed. This bimaterial ambiguity is a strong argument for abandoning the moment density representation of an earthquake and replacing it with a potency density representation, as advocated by Heaton and Heaton (1989) and Ben-Zion (2001). The potency density tensor $p_{k l}=1 / 2 \Delta u\left(n_{k} e_{l}+n_{l} e_{k}\right)$ depends only on the slip $\Delta u_{k}=\Delta u e_{k}$ and is independent of the discontinuous elastic stiffness. The response $u_{n}(\mathbf{r}, t)$ at any point $\mathbf{r}$ and at any time $t$ in the medium is given in terms of the potency density tensor $p_{k l}(\xi, \tau)$ by equation (25). The Green stress tensor $T_{k l n}^{ \pm}(\xi, t ; \mathbf{r}, \tau)$ in equation (25) may be evaluated on either side of the fault, and the result is valid even if the stiffness $C_{i j k l}^{+} \neq C_{i j k l}^{-}$and, therefore, the strain $E_{i j n}^{+} \neq E_{i j n}^{-}$are discontinuous.

\section{Acknowledgments}

This work was supported by National Science Foundation Grants EAR-0105387 and EAR-0126184.

\section{References}

Aki, K. (1966). Generation and propagation of $G$ waves from the Niigata earthquake of June 16, 1964. 2. Estimation of earthquake moment, released energy, and strain-drop from the $\mathrm{G}$ wave spectrum, Bull. Earthquake Res. Inst. Tokyo 44, 23-88.

Aki, K., and P. G. Richards (2002). Quantitative Seismology, Second Ed., University Science Books, Sausalito, California.

Ampuero, J.-P., J.-P. Vilotte, and F.-J. Sánchez-Sesma (2002). Nucleation of rupture under slip dependent friction law: simple models of fault zone, J. Geophys. Res. 107, B12, doi 10.1029/2001JB000452.

Andrews, D. J. (1980). Fault impedance and earthquake energy in the Fourier transform domain, Bull. Seism. Soc. Am. 70, 1683-1698.

Andrews, D. J., and Y. Ben-Zion (1997). Wrinkle-like slip pulse on a fault between different materials, J. Geophys. Res. 102, 553-571. 
Backus, G., and M. Mulcahy (1976a). Moment tensors and other phenomenological descriptions of seismic sources. 1. Continuous displacements, Geophys. J. R. Astr. Soc. 46, 341-361.

Backus, G., and M. Mulcahy (1976b). Moment tensors and other phenomenological descriptions of seismic sources. 2. Discontinuous displacements, Geophys. J. R. Astr. Soc. 47, 301-329.

Ben-Menahem, A., and S. J. Singh (1981). Seismic Waves and Sources, Springer-Verlag, New York

Ben-Zion, Y. (1989). The response of two joined quarter spaces to SH line sources located at the material discontinuity interface, Geophys. $J$. Int. 98, 213-222.

Ben-Zion, Y. (1990). The response of two half spaces to point dislocations at the material interface, Geophys. J. Int. 101, 507-528.

Ben-Zion, Y. (1998). Properties of seismic fault zone waves and their utility for imaging low velocity structures, J. Geophys. Res. 103, 12,56712,585 .

Ben-Zion, Y. (2001). On quantification of the earthquake source, Seism. Res. Lett. 72, 151-152.

Brietzke, G., and Y. Ben-Zion (2003). Examining tendencies of in-plane rupture to migrate to material interfaces (abstract), EOS Trans. AGU 84, no. 46 (Fall Meet. Suppl.), S42C-0190.

Burridge, R., and L. Knopoff (1964). Body force equivalents for seismic dislocations, Bull. Seism. Soc. Am. 54, 1875-1888.

Catchings, R. D., M. J. Rymer, M. R. Goldman, J. A. Hole, R. Huggins, and C. Lippus (2002). High-resolution seismic velocities and shallow structure of the San Andreas fault zone at Middle Mountain, Parkfield, California, Bull. Seism. Soc. Am. 92, 2493-2503.

Chambon, G., J. Schmittbuhl, and A. Corfdir (2002). Laboratory gouge friction: seismic-like slip weakening and secondary rate- and stateeffects, Geophys. Res. Lett. 29, no. 10, doi 10.1029/2001GL014467.

Chester, F. M., J. P. Evans, and R. L. Biegel (1993). Internal structure and weakening mechanisms of the San Andreas fault, J. Geophys. Res. 98, 771-786.

Cochard, A., and J. R. Rice (2000). Fault rupture between dissimilar materials: ill-posedness, regularization, and slip-pulse response, J. Geophys. Res. 105, 25,891-25,907.

Dahlen, F. A., and J. Tromp (1998). Theoretical Global Seismology, Princeton University Press, Princeton, New Jersey.

Eshelby, J. D. (1957). The determination of the elastic field of an ellipsoidal inclusion, and related problems, Proc. R. Soc. London A 241, 376396.

Gilbert, F. (1971). Excitation of the normal modes of the Earth by earthquake sources, Geophys. J. R. Astr. Soc. 22, 223-226.

Got, J. L., J. Fréchet, and F. W. Klein (1994). Deep fault plane geometry inferred from multiplet relative relocation beneath the south flank of Kilauea, J. Geophys. Res. 99, no. B8, 15,375-15,386.

Heaton, T. H., and R. E. Heaton (1989). Static deformations from point forces and force couples located in welded elastic Poissonian halfspaces: implications for seismic moment tensors, Bull. Seism. Soc. Am. 79, 813-841.

Julian, B. R., A. D. Miller, and G. R. Foulger (1998). Non-double-couple earthquakes. 1. Theory, Rev. Geophys. 36, 525-549.

Komatitsch, D., and J. Tromp (1999). Introduction to the spectral element method for three-dimensional wave propagation, Geophys. J. Int. 139, 806-822.

Komatitsch, D., and J. Tromp (2002a). Spectral-element simulations of global seismic wave propagation. I. Validation, Geophys. J. Int. 149, 390-412.

Komatitsch, D., and J. Tromp (2002b). Spectral-element simulations of global seismic wave propagation. II. 3-D models, oceans, rotation, and gravity, Geophys. J. Int. 150, 303-318.

Komatitsch, D., and J.-P. Vilotte (1998). The spectral element method: an effective tool to simulate the seismic response of 2D and 3D geological structures, Bull. Seism. Soc. Am. 88, 368-392.

Kostrov, B. V. (1970). The theory of the focus for tectonic earthquakes, Izv. Bull. Akad. Sci. USSR, Phys. Solid Earth 1, 23-40.
Li, Y. G., J. E. Vidale, K. Aki, and F. Xu (2000). Depth-dependent structure of the Landers fault zone from trapped waves generated by aftershocks, J. Geophys. Res. 105, no. B3, 6237-6254.

McGuire, J. J., L. Zhao, and T. H. Jordan (2002). Predominance of unilateral rupture for a global catalog of large earthquakes, Bull. Seism. Soc. Am. 92, 3309-3317.

Mura, T. (1982). Micromechanics of Defects in Solids, Martinus Nijhoff Publishers, Leiden, The Netherlands.

Nadeau, R. M., and L. R. Johnson (1998). Seismological studies at Parkfield VI: moment release rates and estimates of source parameters for small repeating earthquakes, Bull. Seism. Soc. Am. 88, 790-814.

Ohtani, T., K. Fujimoto, H. Ito, H. Tanaka, N. Tomida, and T. Higuchi (2000). Fault rocks and past to recent fluid characteristics from the borehole survey of the Nojima fault ruptured in the 1995 Kobe earthquake, southwest Japan, J. Geophys. Res. 105, no. B7, 16,161-16,171.

Poliakov, A. N. B., R. Dmowska, and J. R. Rice (2002). Dynamic shear rupture interactions with fault bends and off-axis secondary faulting, J. Geophys. Res. 107, no. B11, doi 10.1029/2001JB000572.

Rubin, A. M. (2002). Aftershocks of microearthquakes as probes of the mechanics of rupture, J. Geophys. Res. 107, no. B7, doi 10.1029/ 2001JB000496.

Rubin, A. M., and D. Gillard (2000). Aftershock asymmetry/rupture directivity among central San Andreas fault microearthquakes, J. Geophys. Res. 105, 19,095-19,109.

Thurber, C., S. Roecker, K. Roberts, M. Gold, L. Powell, and K. Rittger (2003). Earthquake locations and three-dimensional fault zone structure along the creeping section of the San Andreas fault near Parkfield, CA: preparing for SAFOD, Geophys. Res. Lett. 30, no. 3, 1112, doi 10.1029/2002GL016004.

Waldhauser, F., and W. L. Ellsworth (2002). Fault structure and mechanics of the Hayward Fault, California, from double-difference earthquake locations, J. Geophys. Res. 107, no. B3, doi 10.1029/2000JB000084.

Woodhouse, J. H. (1981). The excitation of long-period seismic waves by a source spanning a structural discontinuity, Geophys. Res. Lett. 8 , 1129-1131.

Wu, Z. L., and Y. T. Chen (2003). Definition of seismic moment at a discontinuity interface, Bull. Seism. Soc. Am. 93, 1832-1834.

Yamashita, T. (2000). Generation of microcracks by dynamic shear rupture and its effects on rupture growth and elastic wave radiation, Geophys. J. Int. 143, 395-406.

\section{Appendix: Additional Ambiguities}

The bimaterial ambiguity discussed here is distinct from two other moment tensor ambiguities, which have been noted and discussed previously by Backus and Mulcahy (1976b) and Woodhouse (1981). We review these two additional sources of ambiguity briefly in this appendix.

The first additional ambiguity is one that arises even in the case of slip on a fault $\Sigma$ with no contrast in stiffness, $C_{i j k l}^{+}=C_{i j k l}^{-}$. Backus and Mulcahy (1976b) have noted that it is always possible to augment the moment density tensor $m_{i j}=C_{i j k l} p_{k l}$ on such a fault by adding a force-free density $\phi_{i j}(\xi, \tau)$, satisfying

$$
\iint_{\Sigma} \phi_{i j}(\xi, \tau) \partial_{i} \delta(\mathbf{x}-\xi) d^{2} \xi=0
$$

The constraint (A1) guarantees that the equivalent body force $f_{j}^{*}(\mathbf{x}, \tau)$ and, therefore, the response $u_{n}(\mathbf{r}, t)$ associated with the densities $m_{i j}+\phi_{i j}$ are identical. An arbitrary sym- 
metric tensor field $\phi_{i j}=\phi_{j i}$ on $\Sigma$ can be decomposed in the form

$$
\phi_{i j}=n_{i} n_{i} \phi^{\Sigma}+n_{i} \phi_{j}^{\Sigma}+n_{j} \phi_{i}^{\Sigma}+\phi_{i j}^{\Sigma}
$$

where $n_{i} \phi_{i}^{\Sigma}=0$ and $n_{i} \phi_{i j}^{\Sigma}=n_{j} \phi_{i j}^{\Sigma}=0$. The fields $\phi^{\Sigma}$, $\phi_{j}^{\Sigma}$, and $\phi_{i j}^{\Sigma}=\phi_{j i}^{\Sigma}$ are called the scalar, tangent vector, and tangent tensor parts of $\phi_{i j}$, respectively. When $\phi_{i j}$ is given, the scalar part is found from $\phi^{\Sigma}=n_{i} n_{j} \phi_{i j}$, the tangent vector part is found from $\phi_{j}^{\Sigma}=n_{i} \phi_{i j}-n_{j} \phi^{\Sigma}$, and the tangent tensor part is found by solving equation (A2) for $\phi_{i j}^{\Sigma}$. Backus and Mulcahy (1976b) show that a field $\phi_{i j}$ is force-free in the sense of equation (A1) if and only if

$$
\begin{aligned}
\phi^{\Sigma} & =0 \text { on } \Sigma, \\
\phi_{j}^{\Sigma} & =0 \text { on } \Sigma, \\
\partial_{i}^{\Sigma} \phi_{i j}^{\Sigma} & =0 \text { on } \Sigma, \\
b_{i} \phi_{i j}^{\Sigma} & =0 \text { on } \partial \Sigma,
\end{aligned}
$$

where $\partial_{i}^{\Sigma}=\partial_{i}-n_{i}\left(n_{k} \partial_{k}\right)$, and $b_{i}$ is the unit normal to the boundary $\partial \Sigma$, tangent to $\Sigma$, and pointing out of $\Sigma$. Any field $\phi_{i j}$ satisfying the constraints (A3-A6) can be added to the moment tensor density $m_{i j}=C_{i j k l} p_{k l}$, with no effect on the motion $u_{n}(\mathbf{r}, t)$. The moment density tensor in an isotropic medium $m_{i j}=\mu \Delta u\left(n_{i} e_{j}+n_{j} e_{i}\right)$ satisfies $m^{\Sigma}=0$ and $m_{i j}^{\Sigma}=0$, so no portion of it is force free. This force-free ambiguity does not arise if one adopts a potency density rather than a moment density, or equivalent body force, representation of a finite fault.

The context of the second ambiguity is the determination of the response $u_{n}(\mathbf{r}, t)$ to a smooth strain or stress glut source, in the limit of long-wavelength waves. In that limit, we can approximate the Green strain in equation (18) by

$$
E_{n i j}(\mathbf{r}, t ; \mathbf{x}, \tau) \approx E_{n i j}(\mathbf{r}, t ; \mathbf{s}, \tau),
$$

where $\mathbf{s}$ is some fixed fiducial location of the source. The long-wavelength response can be written in this point-source approximation in the form

$$
u_{n}(\mathbf{r}, t) \approx \int_{0}^{t} E_{n i j}(\mathbf{r}, t ; \mathbf{s}, \tau) M_{i j}(\tau) d \tau,
$$

where

$$
M_{i j}(\tau)=\iiint_{V} \tau_{i j}^{*}(\mathbf{x}, \tau) d^{3} \mathbf{x}
$$

is the moment tensor. In a fault source, this tensor is given by

$$
M_{i j}(\tau)=\iint_{\Sigma} m_{i j}(\xi, \tau) d^{2} \xi
$$

where, for simplicity, we have assumed that there is no contrast in stiffness $C_{i j k l}$ across the fault. The ambiguity arises whenever the source region $V$ or $\Sigma$ spans a welded discontinuity in the medium, such as the Moho. The integrand in equation (A8) becomes in that case $E_{n i j}^{+} M_{i j}^{+}+E_{n i j}^{-} M_{i j}^{-}$, where $E_{n i j}^{ \pm}$are the strains at fiducial points $\mathbf{S}^{ \pm}$on either side of the discontinuity, and

$$
M_{i j}^{ \pm}(\tau)=\iiint_{V^{ \pm}} \tau_{i j}^{*}(\mathbf{x}, \tau) d^{3} \mathbf{x}
$$

or

$$
M_{i j}^{ \pm}(\tau)=\iint_{\Sigma^{ \pm}} m_{i j}^{*}(\xi, \tau) d^{2} \xi
$$

are the corresponding partial moment tensors.

Woodhouse (1981) showed that it is possible to rewrite the response $u_{n}(\mathbf{r}, t)$ solely in terms of the strains $E_{n i j}^{+}$or $E_{n i j}^{-}$on one side or the other:

$$
E_{n i j}^{+} M_{i j}^{+}+E_{n i j}^{-} M_{i j}^{-}=E_{n i j}^{+} \mu_{i j}^{+}=E_{n i j}^{-} \mu_{i j}^{-} .
$$

In an isotropic elastic medium, the plus-side and minus-side moment tensors $\mu_{i j}^{ \pm}$are given by (Dahlen and Tromp, 1998, section 5.4.6):

$$
\begin{aligned}
& \mu_{x x}^{ \pm}=M_{x x}^{+}+M_{x x}^{-}+a^{ \pm} M_{z z}^{\mp}, \\
& \mu_{y y}^{ \pm}=M_{y y}^{+}+M_{y y}^{-}+a^{ \pm} M_{z z}^{\mp}, \\
& \mu_{z z}^{ \pm}=M_{z z}^{ \pm}+b^{ \pm} M_{z z}^{\mp}, \\
& \mu_{x z}^{ \pm}=M_{x z}^{ \pm}+c^{ \pm} M_{x z}^{\mp}, \\
& \mu_{y z}^{ \pm}=M_{y z}^{ \pm}+c^{ \pm} M_{y z}^{\mp}, \\
& \mu_{x y}^{ \pm}=M_{x y}^{+}+M_{x y}^{-},
\end{aligned}
$$

where $\hat{\mathbf{z}}$ is the unit normal to the discontinuity, and where

$$
a^{ \pm}=\frac{\left(\kappa^{ \pm}-\frac{2}{3} \mu^{ \pm}\right)-\left(\kappa^{\mp}-\frac{2}{3} \mu^{\mp}\right)}{\kappa^{\mp}+\frac{4}{3} \mu^{\mp}}
$$

$$
b^{ \pm}=\frac{\kappa^{ \pm}+\frac{4}{3} \mu^{ \pm}}{\kappa^{\mp}+\frac{4}{3} \mu^{\mp}}, \quad c^{ \pm}=\frac{\mu^{ \pm}}{\mu^{\bar{\mp}}} .
$$

Evidently, the true long-wavelength source is equivalent to a moment tensor $\mu_{i j}^{+}$placed on the plus side or to a moment tensor $M_{i j}^{-}$placed on the minus side of the discontinuity. Neither $M_{i j}^{+}$nor $M_{i j}^{-}$represents the true moment tensor, 
which is given by $M_{i j}=M_{i j}^{+}+M_{i j}^{-}$. The two apparent moment tensors $\mu_{i j}^{+}$and $\mu_{i j}^{-}$are the source mechanisms that would be obtained from an inversion of observed seismograms $u_{n}(\mathbf{r}, t)$ under the assumption that the earthquake is situated at a point on either the plus or the minus side of the discontinuity.

The plus-side and minus-side mechanisms $\mu_{i j}^{+}$and $\mu_{i j}^{-}$need not have the same orientation, but if they do, then it is possible to write

$$
\mu_{i j}^{+}=\gamma \mu_{i j}^{\gamma}, \quad \mu_{i j}^{-}=(1-\gamma) \mu_{i j}^{\gamma},
$$

where $\mu_{i j}^{Y}$ is "the" moment tensor under the same-orientation assumption (A22), and $0 \leq \gamma \leq 1$ is a parameter specifying the fraction of the moment lying on the plus side of the discontinuity. Woodhouse (1981) shows how to find $M_{i j}^{p}$ in terms of the observables $\mu_{i j}^{+}$and $\mu_{i j}^{-}$; however, there are an infinite number of such moment tensors, depending on the choice of the parameter $\gamma$. Julian et al. (1998) illustrated how this ambiguity perturbs the inversion of earthquake moment tensors, introducing distorsion of the orientation and seismic moment and apparent non-double-couple components. This "which side of the discontinuity" ambiguity of an earthquake in the long-wavelength approximation is reminiscent of the "which side of the fault" ambiguity of an earthquake characterized by faulting on a finite bimaterial interface.

Unlike the bimaterial ambiguity, the ambiguity of a point source situated on a discontinuity cannot be eliminated by switching to a potency rather than a moment representation. To obtain such a point-source potency representation, we approximate the Green stress in equation (19) by

$$
T_{k l n}(\mathbf{x}, t ; \mathbf{r}, \tau) \approx T_{k l n}(\mathbf{s}, t ; \mathbf{r}, \tau)
$$

and write the long-wavelength response in the form

$$
u_{n}(\mathbf{r}, t) \approx \int_{0}^{t} T_{k l n}(\mathbf{s}, t ; \mathbf{r}, \tau) P_{k l}(\tau) d \tau,
$$

where either

$$
P_{k l}(\tau)=\iiint_{V} \varepsilon_{k l}^{*}(\mathbf{x}, \tau) d^{3} \mathbf{x}
$$

or

$$
P_{k l}(\tau)=\iint_{\Sigma} p_{k l}(\xi, \tau) d^{2} \xi
$$

is the potency tensor. If the source region $V$ or $\Sigma$ spans a welded discontinuity, then the integrand in equation (A24) is replaced by $T_{k l n}^{+} P_{k l}^{+}+T_{k l n}^{-} P_{k l}^{-}$, where

$$
P_{k l}^{ \pm}(\tau)=\iiint_{V^{ \pm}} \varepsilon_{k l}^{*}(\mathbf{x}, \tau) d^{3} \mathbf{x}
$$

or

$$
P_{k l}^{ \pm}(\tau)=\iint_{\Sigma^{ \pm}} p_{k l}(\xi, \tau) d^{2} \xi
$$

are partial potency tensors analogous to the partial moment tensors $M_{i j}^{ \pm}(\tau)$ in equations (A11 and A12).

As in the moment tensor case, it is possible to rewrite the response $u_{n}(\mathbf{r}, t)$ in terms of potency tensors $\Phi_{k l}^{+}$and $\Phi_{k l}^{-}$corresponding to a source that is situated entirely on the plus or the minus side of the discontinuity:

$$
T_{k l n}^{+} P_{k l}^{+}+T_{k l n}^{-} P_{k l}^{-}=T_{k l n}^{+} \Phi_{k l}^{+}=T_{k l n}^{-} \Phi_{k l}^{-} .
$$

Stress and strain continuity considerations analogous to those used by Woodhouse (1981) can be used to show that, in an isotropic elastic medium, the plus-side and minus-side potency tensors are given explicitly by

$$
\begin{aligned}
& \Phi_{x x}^{ \pm}=P_{x x}^{ \pm}+d^{ \pm} P_{x x}^{\mp}+e^{ \pm} P_{y y}^{\mp}, \\
& \Phi_{y y}^{ \pm}=P_{y y}^{ \pm}+d^{ \pm} P_{y y}^{\mp}+e^{ \pm} P_{x x}^{\mp}, \\
& \Phi_{z z}^{ \pm}=P_{z z}^{+}+P_{z z}^{-}+f^{ \pm}\left(P_{x x}^{\mp}+P_{y y}^{\mp}\right), \\
& \Phi_{x z}^{ \pm}=P_{x z}^{+}+P_{x z}^{-}, \\
& \Phi_{y z}^{ \pm}=P_{y z}^{+}+P_{y z}^{-}, \\
& \Phi_{x y}^{ \pm}=P_{x y}^{ \pm}+c^{\mp} P_{x y}^{\mp},
\end{aligned}
$$

where

$$
d^{ \pm}=\frac{1+\frac{2}{3}\left(\frac{\mu^{ \pm}}{\kappa^{ \pm}}+\frac{\mu^{\mp}}{\kappa^{\mp}}\right)}{\mu^{ \pm}\left(\frac{4}{3 \kappa^{\mp}}+\frac{1}{\mu^{\mp}}\right)}
$$

$$
e^{ \pm}=\frac{\frac{2}{3}\left(\frac{\mu^{ \pm}}{\kappa^{ \pm}}-\frac{\mu^{\mp}}{\kappa^{\mp}}\right)}{\mu^{ \pm}\left(\frac{4}{3 \kappa^{\mp}}+\frac{1}{\mu^{\mp}}\right)}
$$

$$
f^{ \pm}=\frac{\left(\frac{2}{3 \kappa^{ \pm}}-\frac{1}{\mu^{ \pm}}\right)-\left(\frac{2}{3 \kappa^{\mp}}-\frac{1}{\mu^{\mp}}\right)}{\frac{4}{3 \kappa^{\mp}}+\frac{1}{\mu^{\mp}}}
$$

It is evident that neither $\Phi_{k l}^{+}$nor $\Phi_{k l}^{-}$is equal to the true potency tensor, which is given by $P_{k l}=P_{k l}^{+}+P_{k l}^{-}$. If one 
makes the assumption that the observables $\Phi_{k l}^{+}$and $\Phi_{k l}^{-}$have the same orientation, so that

$$
\Phi_{k l}^{+}=\gamma \Phi_{k l}^{\gamma}, \quad \Phi_{k l}^{-}=(1-\gamma) \Phi_{k l}^{\gamma},
$$

then it is possible to find an infinite suite of possible potency tensors $\Phi_{k l}^{\gamma}$, where $0 \leq \gamma \leq 1$ is now a measure of the fraction of the potency that lies on the plus side of the fault. The only components of $M_{i j}$ and $P_{k l}$ that can be determined unambiguously are $M_{x y}, M_{x x}-M_{y y}$, and $P_{x z}, P_{y z}$. The ambiguity of both $M_{i j}$ and $P_{k l}$ are artifacts of the point-source approximation (A23). The finite-fault representation (25) of the motion $u_{n}(\mathbf{r}, t)$ is valid even in the case of $\operatorname{slip} \Delta u_{k}(\xi, \tau)$ on a bimaterial interface that cuts obliquely across another discontinuity, such as the Moho.

Department of Geosciences

Princeton University

Princeton, New Jersey 08544

(J.-P.A.)

Manuscript received 27 May 2004. 